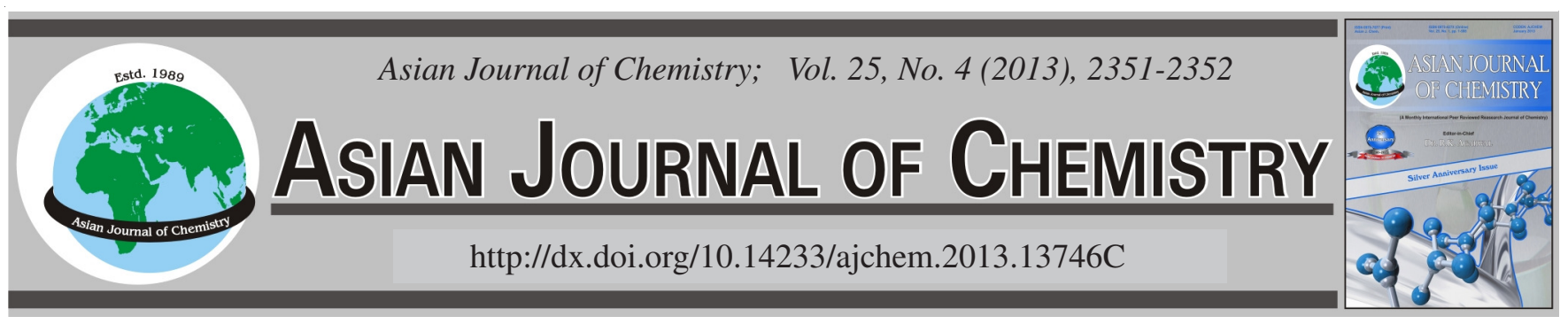

NOTE

\title{
Urothermal Synthesis, Structure of One 3-Dimensional Neodymium(III) Coordination Polymer
}

\author{
ZHIQIANG JIANG
}

Biology and Chemistry Engineering College of Panzhihua University, Sichuan 617000, P.R. China

Corresponding author: E-mail: myjiangzq@163.com

(Received: 17 March 2012;

Accepted: 31 October 2012)

AJC-12356

\begin{abstract}
A 3D neodymium(III) coordination polymer was synthesized via urothermal method and characterized by X-ray diffraction. This compound crystallized in the triclinic space group P-1 with $\mathrm{a}=9.8303(3) \AA, \mathrm{b}=18.8077(7) \AA, \mathrm{c}=19.1199(8) \AA, \alpha=96.577(3)^{\circ}, \beta=$ $99.509(3)^{\circ}, \gamma=90.295(3)^{\circ}, V=3462.4(2) \AA^{3}, Z=2$.
\end{abstract}

Key Words: Urothermal synthesis, Structure, Neodymium(III) coordination polymer.

ᄂ ------------------------------$\lrcorner$

The synthesis of three-dimensional (3D) coordination polymers is still a matter of high concern owing to controlled design-synthesis such as different pore size, shape, multifarious topology and tunable host-guest interactions ${ }^{1-5}$. Solvent plays an important role in the self-assembly of coordination polymers. The reversible binding of urea derivatives to metal sites and urea derivatives acted as the structure-director are an interesting aspect of urothermal synthetic process. Such urothermal method is well suited for the creation of coordination polymers materials with active unsaturated metal centers and numerous new materials are created with large variety of urea derivatives, various organic ligands and metal. In this work, we have constructed a 3D neodymium(III) coordination polymer using this urothermal method.

All reagents and solvents employed were commercially available and used as received without further purification.

Preparation of compound: 3-Dimensional neodymium(III) coordination polymer was synthesized from an urothermal reaction. Terephthalic acid $\left(\mathrm{H}_{2} \mathrm{bdc}, 0.0515 \mathrm{~g}, 0.31 \mathrm{mmol}\right)$, $\mathrm{Nd}\left(\mathrm{NO}_{3}\right)_{3} \cdot 6 \mathrm{H}_{2} \mathrm{O}(0.0791 \mathrm{~g}, 0.18 \mathrm{mmol}), 1,3$-dimethyl-2imidazolidone (DMI, $3 \mathrm{~g}, 26.3 \mathrm{mmol}$ ) and urea (1.9103 g, 15 mmol) were mixed and then placed in a Parr Teflon-lined stainless steel vessels. After that these vessels were capped and heated at $120^{\circ} \mathrm{C}$ for 3 days. The autoclave was subsequently allowed to cool to room temperature. The resulting colourless single crystals $\left[\mathrm{Nd}_{4}\left(\mathrm{H}_{2} \mathrm{bdc}\right)_{6}(\mathrm{DMI})_{4}\right]$ (1) were washed by acetone.

X-crystallography: Suitable single crystals of compound $1\left[\mathrm{Nd}_{4}\left(\mathrm{H}_{2} \mathrm{bdc}\right)_{6}(\mathrm{DMI})_{4}\right]$ were carefully selected under an optical microscope and glued to thin glass fibers. Whereafter, single-crystal X-ray diffraction analyses were performed on a computer-controlled XCalibur E CCD diffractometer with graphite-monochromated $\mathrm{MoK}_{\alpha}$ radiation $\left(\lambda_{\mathrm{MoK} \alpha}=0.71073 \AA\right)$ at $\mathrm{T}=293.2 \mathrm{~K}$. Empirical absorption corrections were made using the SADABS program ${ }^{6}$. The structures were solved using the direct method and refined by full-matrix least-squares methods on $\mathrm{F}^{2}$ by using the SHELX-97 program package ${ }^{7}$. All non-hydrogen atoms were refined anisotropically and hydrogen atoms attached to carbon or nitrogen atoms were fixed at their ideal positions.

Structure description: Single crystal X-ray analysis of compound 1 showed unique three-dimensional (3D) open frameworks consisting of four $\mathrm{Nd}(\mathrm{III})$ ions, six $\mathrm{H}_{2}$ bdc ligands and 4-DMI molecule (Fig. 1a). The four metal centers showed the same configuration. Each $\mathrm{Nd}(\mathrm{III})$ is primarily coordinated to six oxygen atoms from the carboxylic groups of one and half $\mathrm{H}_{2}$ bdc ligand and one oxygen atom from DMI molecule. Each pair of $\mathrm{Nd}(\mathrm{III})$ are re joined by $\mathrm{H}_{2}$ bdc liagands adopting a $\mu_{2}$-bridging coordination fashion with two bis-monodentate carboxylate groups to result in one-dimensional (1D) chains (Fig. 1b). These one-dimensional chains are further interlinked by the organic $\mathrm{H}_{2}$ bdc bridges to produce two-dimensional networks (Fig. 1c). These two-dimensional layers coordinate to the carboxylic groups from $\mathrm{H}_{2}$ bdc ligands to complete the construction of the 3D framework (Fig.1d).

\section{REFERENCES}

1. B.F. Hoskins and R. Robson, J. Am. Chem. Soc., 111, 5962 (1989).

2. S. Kitagawa, R. Kitaura and S.I. Noro, Angew. Chem. Int. Ed., 43, 2334 (2004).

3. G. Ferey, Chem. Soc. Rev., 37, 191 (2008)

4. J.R. Long and O.M. Yaghi, Chem. Soc. Rev., 38, 1213 (2009). 
(a)

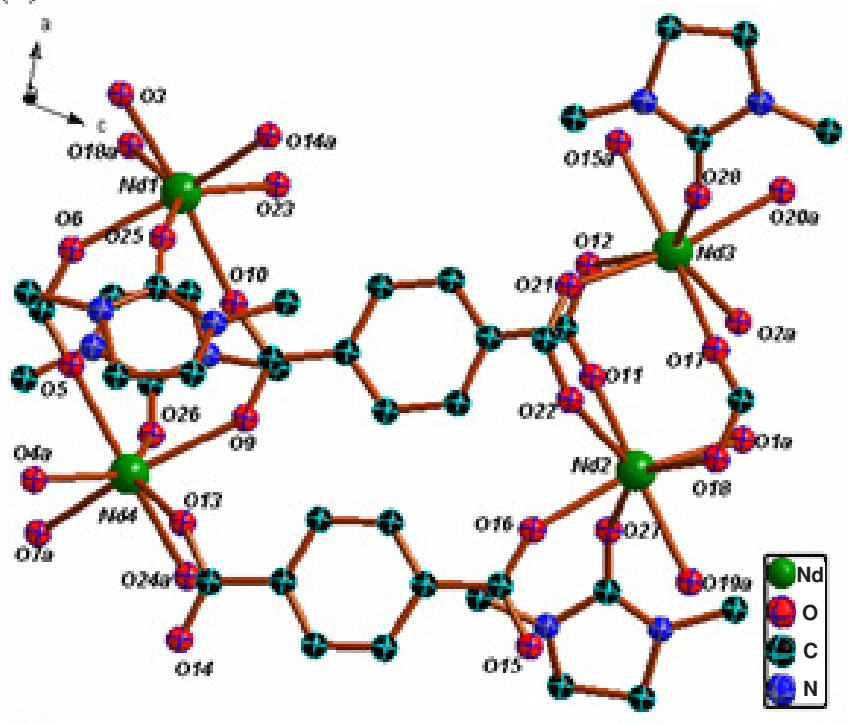

(b) (c)

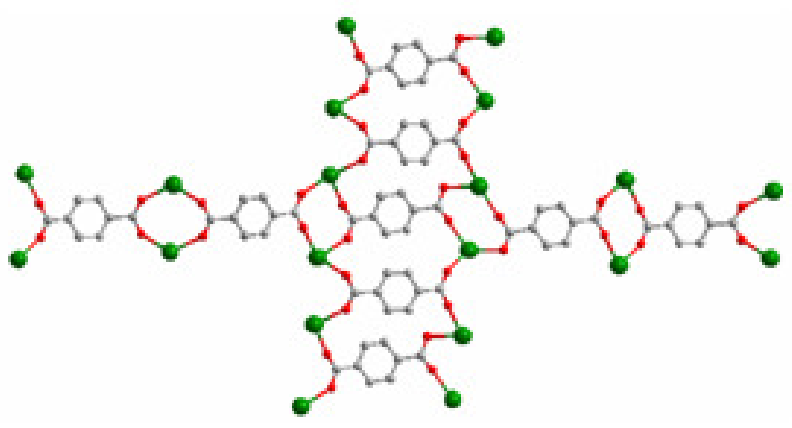

(d)

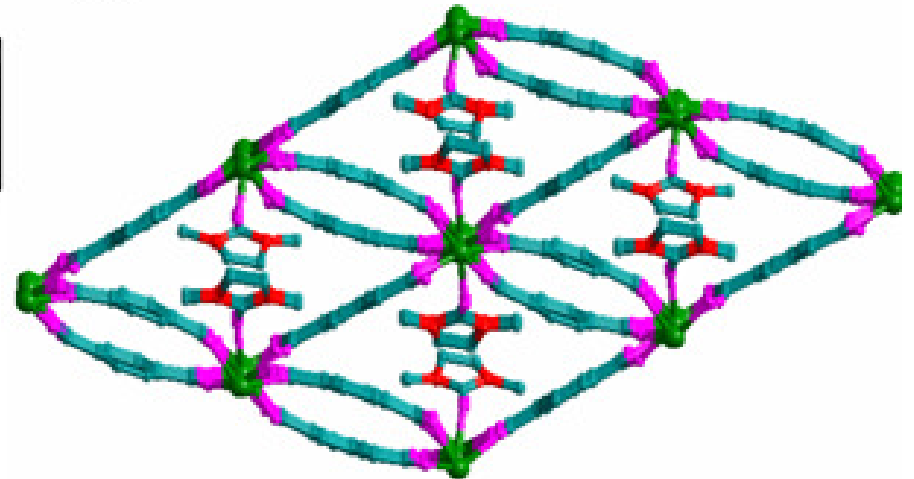

Fig. 1. (a) Coordination envionment of compound 1; (b) One-dimensional chain of compound 1; (c) Two-dimensional network in compound 1; (d) View of the three-dimensional architecture of compound $\mathbf{1}$

5. H.X. Deng, C.J. Doonan, H. Furukawa, R.B. Ferreira, J. Towne, C.B. Knobler, B. Wang and O.M. Yaghi, Science, 327, 846 (2010).

6. G.M. Sheldrick, SADABS, Program for Area Detector Adsorption Correction; Institute for Inorganic Chemistry, University of Göttingen: Göttingen, Germany (1996).
7. G.M. Sheldrick, SHELXL-97, Program for Solution of Crystal Structures; University of Göttingen: Göttingen, Germany (1997). 